\title{
Intestinal microbiota analysis supports inclusion of gluten-free oats to diet of subjects with celiac disease or gluten sensitivity
}

\section{Abstract}

Gluten-related disorders form the umbrella term for all conditions related to gluten ingestion, such as celiac disease (CeD) and nonceliac gluten sensitivity (NCGS). A life-long exclusion of gluten from diet is currently the only effective treatment in remitting the symptoms of these diseases. However, a life-long, strict GFD is challenging to maintain due to social and economic burdens. In addition, it may lead to restricted and nutritionally suboptimal diet. Thus, the possibility of using nutritious and fiber-rich oats would diversify the GFD, improving general palatability, sensory properties such as texture and fiber-content of the diet. Pure oats are being grown and produced following strict agricultural practices to minimize any contamination with other cereals. Indeed, consumption of pure, uncontaminated oats would allow a wider selection of foods for individuals with gluten related disorders. However, the inclusion of oats into GFD is not globally applied yet. The aim of this study was to evaluate the effect of daily consumption of oat products on intestinal microbiota composition and activity in subjects with gluten-related disorders. Subjects with CeD $(n=19)$ or NCGS $(n=10)$ and healthy volunteers $(n=14)$ were recruited to the study. Study subjects completed food diaries for four days preceding fecal sample collection. Intestinal microbiota composition was characterized using 16S MiSeq sequencing. The total energy intake was comparable between the study groups. However, NCGS subjects consumed more energy (E \%) from protein and less from carbohydrates when compared to healthy controls $(p=0.025$ and $p=0.045$, respectively). Dietary fiber intake was relatively high in all subjects and no differences were observed between the study groups $(\mathrm{p}=0.79)$. Total microbiota profiles were comparable between CeD, NCGS and healthy controls. In addition, no differences were observed in microbiota richness or diversity between the study groups. Phylum-level microbial abundances exhibited high inter-individual variation, but did not differ between the study groups. To conclude, microbiota markers demonstrated that oat consumption was safe and beneficial for subjects with CeD or NCGS. Oat consumption was shown to enrich available gluten-free diets and to increase dietary fiber intake to the recommended levels.

\section{Conflict of Interest}

There is no conflict of interest 